\title{
X-ray Measurements of the Depth Dependence of Stress in Gold Films
}

\author{
S. Brennan*, A. Munkholm**, O.S. Leung ${ }^{\dagger}$, W.D. Nix ${ }^{\dagger}$ \\ * Stanford Synchrotron Radiation Laboratory, Stanford Linear Accelerator Center, Stanford, CA 94309 \\ ** Chemistry Division, Argonne National Laboratory, Argonne, IL 60439 \\ $\dagger$ Dept. Materials Science and Engineering, Stanford University, Stanford, CA 94305 \\ (September 21, 1999) \\ $\mathrm{X}$-rays are used to determine the stress as a function of depth for five evaporated gold films of 0.8-2.5 \\ microns thickness. The depth dependence is achieved by varying the incident angle of the $\mathrm{x}$-rays, \\ which effects the penetration depth of the x-rays into the film. The films, which have a different \\ thermal expansion coefficient than the silicon substrate, are strained as a result of thermal cycling \\ after deposition. We find essentially no variation with stress as a function of depth for these films.
}

\section{A. Introduction}

Residual stress in metal films deposited on silicon substrates is an important topic from both a phenomenological and technological standpoint. Thermal cycling after deposition results in residual elastic strain due to differential thermal expansion between the film and the silicon substrate. Gold on silicon is a model system for understanding mechanisms of dislocation motion in thin films, and is also of interest to the integrated circuit community due to the effect of residual stress on interconnect failure processes. Wafer curvature techniques can be used to determine the overall residual stress in these films, but are unable to resolve whether the stress varies with depth. By changing the effective penetration depth of an x-ray beam one can determine stress both near the surface and further into the bulk of the film.

Bulk materials properties do not properly predict the strength of materials on short length scales. Recent experimental work studying torsion in thin wires [1], nanoindentation [2] and microbending of thin plates [3] all suggest that materials deformed on the micron scale are stronger than predicted. Thin films are specific examples of increased strength with shorter length scales. Both wafer curvature and $\mathrm{x}$-ray techniques have shown that the strength of films are approximately inversely proportional to thickness in a variety of materials $[4,5]$. Nix [6] has proposed that the energy of dislocation segments deposited along film interfaces can explain the increase in strength with decreasing thickness. Since the grain structure of thin metal films tends to be columnar with lateral dimensions comparable to the thickness, a similar mechanism could be occurring at grain boundaries [7] which would also have an inverse dependence on thickness. An alternative strengthening mechanism for films on substrates with thicknesses in the micron range is strain gradient plasticity [8]. In this model, the constraint of the substrate or surface oxide causes dislocation pileup [9] at the film/substrate and/or film/oxide interface. These pileups result in strain gradients which may strengthen the film and would be observable with x-rays.

We have studied five gold films evaporated on silicon with either a titanium or a tungsten bonding layer. The film thicknesses range from 0.8 to 2.5 microns. Although less technologically relevant than aluminum or copper, gold has the advantage of not forming a significant surface oxide, which is suspected to play a role in resisting strain relaxation in these films. However, gold is strongly absorbing, which makes it difficult for $\mathrm{x}$-rays to reach the interface in thick films and has required us to employ unusual techniques for measuring the strain as a function of depth in these films.

\section{B. Experimental}

Samples were prepared by coating both sides of 100 $\mathrm{mm}$ diameter, [100] oriented Si wafers with a low-stress $\mathrm{Si}_{3} \mathrm{~N}_{4}$ film. As wafer curvature measurements were also performed on these samples, both sides were coated so that the wafers would not exhibit wafer curvature prior to the deposition of the gold film. An adhesion layer of $100 \AA$ of either $\mathrm{W}$ or Ti was deposited on one side, and gold films of thicknesses between 0.8 and $2.5 \mu \mathrm{m}$ were evaporated onto the wafers. The samples were then thermally cycled between room temperature and $600 \mathrm{degC}$ in an ambient atmosphere. Heating and cooling rates were $\sim 5 \mathrm{degC} / \mathrm{min}$. To confirm that the gold films were not oxidized, x-ray photoelectron spectroscopy (XPS) measurements were performed on two samples. For the $1.75 \mu \mathrm{m}$ sample (deposited on W), the XPS profile showed a small $(\sim 14 \%)$ oxygen signal and a $(\sim 40 \%)$ carbon signal before sputtering. After $\sim 40 \AA$ were removed the oxygen and carbon signals had disappeared. Similar results were obtained for the $0.79 \mu \mathrm{m}$ film deposited on Ti.

Due to the strong absorption of $x$-rays in gold, the method developed by Doerner and Brennan [10] for studying aluminum films could not be used. In that method, the scattering vector lies nearly in the plane of the surface, sampling $\{220\}$ planes perpendicular to the [111] growth direction. Varying the angle of incidence of the $x$-ray beam over a small $(<2 \mathrm{deg})$ range results in large changes in the penetration depth into the film with little change in the direction of the scattering 


\section{DISCLAIMER}

This report was prepared as an account of work sponsored by an agency of the United States Government. Neither the United States Government nor any agency thereof, nor any of their employees, make any warranty, express or implied, or assumes any legal liability or responsibility for the accuracy, completeness, or usefulness of any information, apparatus, product, or process disclosed, or represents that its use would not infringe privately owned rights. Reference herein to any specific commercial product, process, or service by trade name, trademark, manufacturer, or otherwise does not necessarily constitute or imply its endorsement, recommendation, or favoring by the United States Government or any agency thereof. The views and opinions of authors expressed herein do not necessarily state or reflect those of the United States Government or any agency thereof. 


\section{DISCLAIMER}

\section{Portions of this document may be illegible in electronic image products. Images are produced from the best available original document.}


vector. To penetrate to the interface of a $2.5 \mu \mathrm{m}$ gold film, however, an angle of incidence of over 20 degrees is needed, and the scattering vector is no longer approximately parallel to the surface. Because evaporated gold is also highly textured with the [111] direction along the growth direction, tilting the grazing angle up to 20 degrees rotates the scattering vector away from the $\{220\}$ planes, resulting in a signal only from those planes which are not part of the majority texture. Thus a variation on a measurement technique described by Mochrie [11] was employed. In this geometry, the scattering vector is positioned on an off-specular reflection which is part of the majority texture, e.g. the $\{331\}$ or $\{311\}$ planes, which are tilted with respect to the [111] surface of the film. One then rotates the sample about the scattering vector while keeping it on that reflection, as one would do in the " $\psi$ " mode described by Busing and Levy [12] (which is not the $\Psi$-angle used in the rest of this paper). Figure 1 is a schematic of this geometry, with the $\Psi$ angle between the plane normal and the surface normal, the incident $(\alpha)$, and takeoff $(\beta)$ angles. Rotation about the reciprocal lattice vector results in smaller incident and larger take-off angles, which decreases the x-ray penetration depth. The maximum penetration depth occurs with equal incident and take-off angles, as they would be in the " $\Omega=0$ " mode of Busing and Levy. This new geometry is especially advantageous for polycrystalline films because the same ensemble of grains is measured at several different angles of incidence, which is not the case for the geometry used by Doerner and Brennan.

The x-ray experiments were performed at the BESSRC undulator beamline 12-ID-B at the Advanced Photon Source at Argonne National Laboratory. Photons of $28020 \mathrm{eV}$ were transmitted by the monochromator and

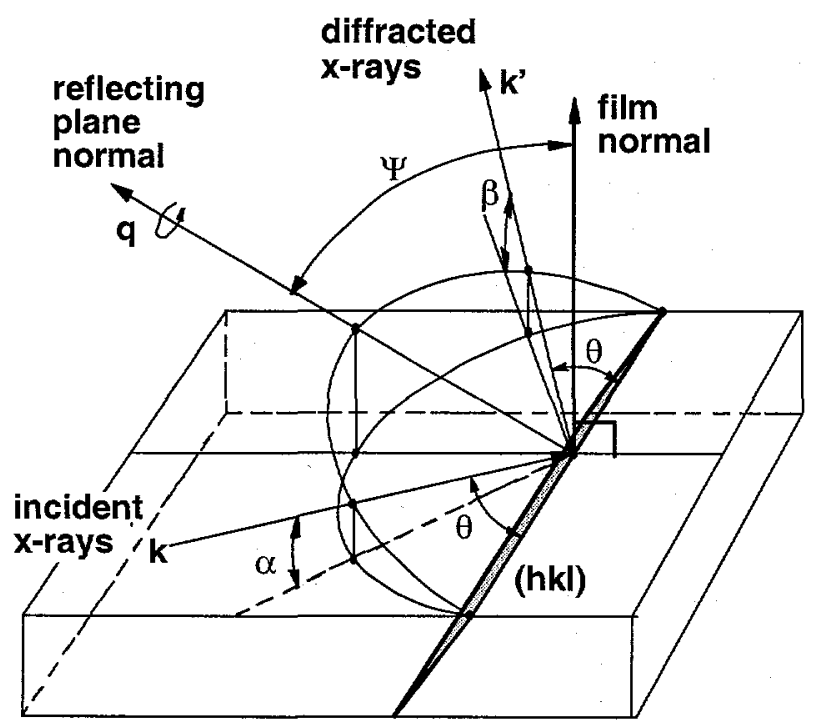

FIG. 1. Scattering geometry showing $\Psi$, the incident $(\alpha)$, and takeoff $(\beta)$ angles. Rotating about $q$ decreases $\alpha$, increases $\beta$, and decreases the $\mathrm{x}$-ray penetration depth. restricted to $250 \times 250 \mu \mathrm{m}$ before striking the sample. The high energy of the photons was necessary to get sufficient penetration into the gold sample. In the results described below, penetration depths will be quoted which are significantly larger than the actual film thickness. In the limiting case of infinite penetration depth, the electric field intensity would be unity throughout the film, and the reported stress and unstrained a 0 would be a simple average over the entire film. For finite penetration depths greater than the film thickness, the average is still over the entire film, but is non-linear, because the electric field intensity is decaying with depth. A result quoted for a penetration depth equal to a film thickness means that roughly $1 / 3^{r d}$ the intensity is from crystallites at that depth, the rest from shallower depths.

The goal of the study is to determine the biaxial stress within the films as a function of depth. Because the $x$ rays are scattering from all depths up to the penetration depth the reported stress for a particular depth is the integral of the stress from the surface to that depth. Also, the penetration depth reported in the results below is the depth into the sample such that the diffracted beam from that depth, exiting the sample and striking the detector, would be $1 / \mathrm{e}$ of the incident intensity (assuming unity for the scattering process). This is different from the normal definition of a penetration depth, which is the depth at which 1 /e of the incident intensity remains. The standard $d_{h k l}$ vs. $\sin ^{2}(\Psi)$ method [13] is used to determine both the biaxial stress and the unstrained lattice parameter a0 for the films. As the same reflections are measured at several penetration depths, depth-dependent analyses can be performed, resulting in stress and unstrained a0 values which are depth-dependent.

\section{Results}

There are several experimental corrections which become necessary prior to analysing the data. Toney and Brennan [14] showed that for a deposited thin film there is an index of refraction correction necessary when the angle of incidence is not equal to the take-off angle of the diffracted beam. This correction is well characterized. However, if the film is rough, the correction is not simply described. There is also a height-error correction which becomes more pronounced as the angle of incidence becomes smaller. Both of these corrections result in the $2 \Theta$ angle becoming larger at smaller angles of incidence. Fortuitously, the (33ī) reflection for [111]-textured gold is unaffected by a biaxial stress, as it tilts in the direction where the strained and unstrained ellipses intersect. The angle at which the two ellipses intersect is dependent on the materials constants of the film. Thus any variation in $2 \Theta$ angle with incident angle for the (33i) reflection must be experimentally induced. 


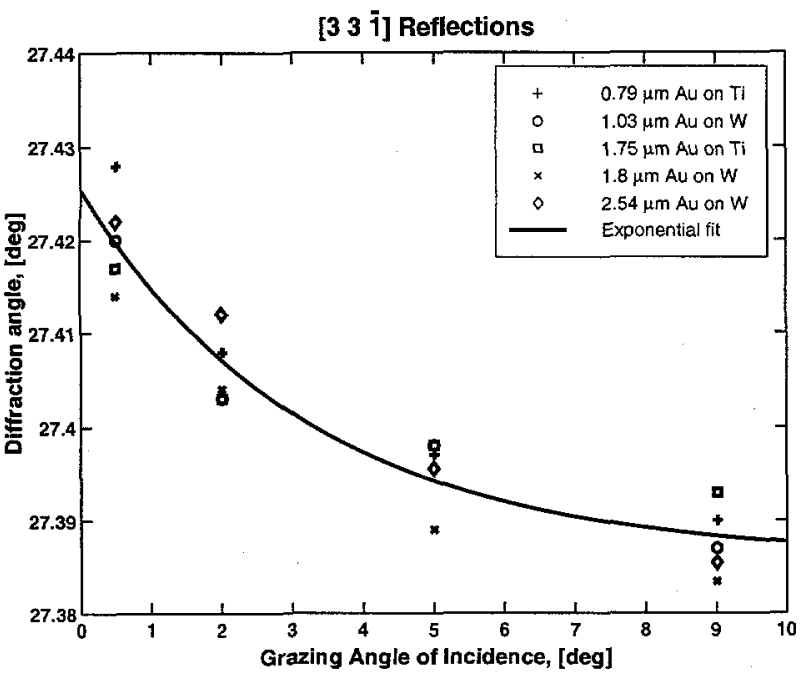

FIG. 2. Diffracted peak positions of the (33ī) reflection for all 5 gold films and an exponential fit to those peak positions vs. angle of incidence.

Figure 2 is a plot of the diffraction angle for the (33i) reflections for all 5 films, as well as an empiricallydetermined exponential fit to the data. The inverse of this exponential fit is used to normalize each of the measured $2 \Theta$ values. Because reflections other than the $\{331\}$ were also measured, the exponential fit is normalized to unity at high angle of incidence prior to correcting the other $2 \Theta$ values. At large angles of incidence there is little change between the corrected and uncorrected data, but the correction is more significant at low angles where the correction is as large as $0.034 \mathrm{deg}$ at a grazing angle of $0.5 \mathrm{deg}$.

Once the $2 \Theta$ values have been corrected, the stress and unstrained $\mathrm{a} 0$ can be extracted. The analysis is done separately for each film. For the analysis, 7 overlapping depth ranges are used: $0 \Rightarrow 0.5 \mu \mathrm{m}, 0.25 \Rightarrow 0.75 \mu \mathrm{m}$, $0.5 \Rightarrow 1.0 \mu \mathrm{m}, 0.75 \Rightarrow 1.25 \mu \mathrm{m}, 1.0 \Rightarrow 1.5 \mu \mathrm{m}, 1.25 \Rightarrow$ $1.75 \mu \mathrm{m}$ and $>1.5 \mu \mathrm{m}$. This results in between 7 and 9 data points per range, and the overlap results in averaging between neighboring ranges. Several points stand out in Figure 3. For 4 of the 5 films, the unstrained a0 averaged over the entire film (the $>1.5 \mu \mathrm{m}$ data) show a remarkably consistent value, being within $0.0003 \AA$ of each other. The $1.75 \mu \mathrm{m}$ Au on Ti film is off by $\sim 0.001 \AA$. There is more spread in the data for the lower penetration depths, which may be in part because of the lower scattering volume, which increases the effective error for those data. Generally, all but a few points are within the $\pm 0.0008 \AA$ error bars. These were derived by using the spread in $2 \Theta$ values for the $(33 \overline{1})( \pm 0.005 \mathrm{deg})$ to determine the spread in unstrained $\mathrm{a} 0$ and stress (for Figure 4).

The biaxial stress for the films is presented in Figure 4, again for the same range of penetration depths as shown in Figure 3. In addition to the stress at each penetration

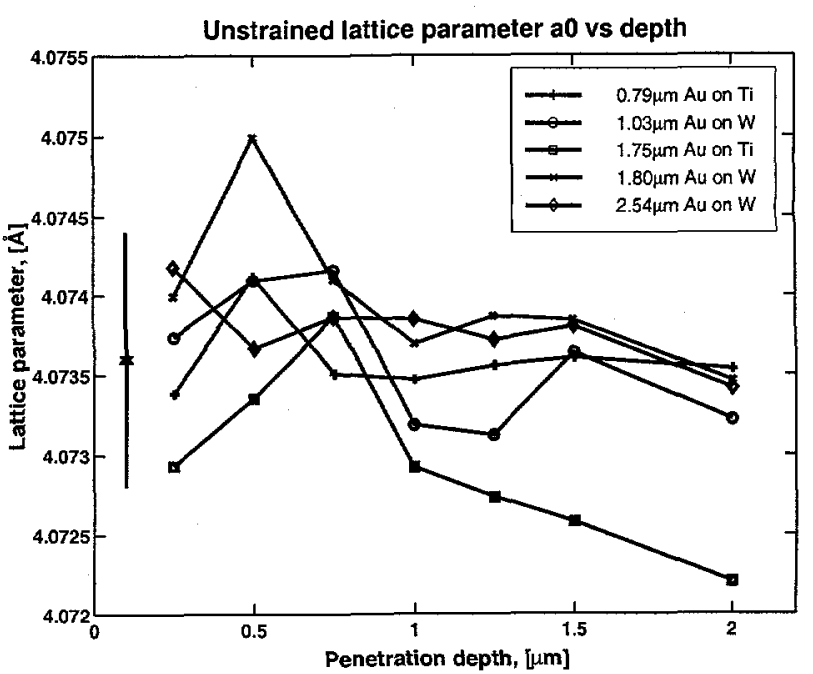

FIG. 3. Unstrained lattice parameter $\mathrm{a} 0$ for 7 overlapping depths for all 5 films. The unstrained $a 0$ is determined from the $d_{h k b}$ vs. $\sin ^{2}(\Psi)$ analysis at a $\Psi$ value based on the specific materials properties of the film. For gold, this angle is $48.14 \mathrm{deg}$. The measurement error of $\pm 0.0008 \AA$ is shown along the lefthand edge as a vertical line.

depth, a linear fit to those data is shown, as the simplest model of whether there is a trend in the stress as a function of penetration depth. For the stress data, the $1.75 \mu \mathrm{m}$ film is significantly different from the others, showing a large depth dependence. The other four films show an average change of $14 \mathrm{MPa}$ between the shallowest and deepest measurements. This change is just larger than our calculated error bars of $\pm 10 \mathrm{MPa}$, which are not shown in Figure 4 because they would be only slightly larger than the markers in the figure. Given that the actual scatter of the stress data with penetration depth is significantly larger than the calculated error, we conclude that there is not a significant variation of stress with depth. The stress for the $1.75 \mu \mathrm{m}$ film decreases by $79 \mathrm{MPa}$ over the same depth range, which is significantly larger than either the error bars or the data scatter. Since this film was fabricated identically to the others, and shows a significantly different unstrained a0, we suspect an unknown factor is causing both the stress variation with depth and the different unstrained $\mathrm{a} 0$ for this film. There are other points to be noted about the films. Both films which have gold deposited on a titanium bonding layer $(0.79$ and $1.75 \mu \mathrm{m})$ show larger biaxial stresses than those deposited on tungsten. It is unclear how the bonding layer affects the residual stress. The highest penetration-depth data for all the films show a picture consistent with the wafer curvature technique, which is that thinner films tend to have higher residual stresses, although the bonding layer clearly plays a significant role. 


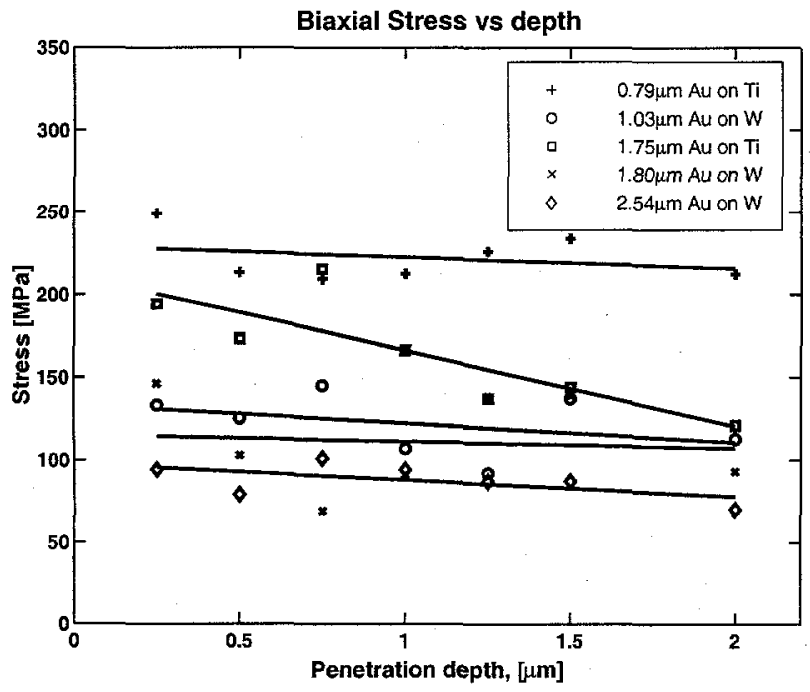

FIG. 4. Stress vs. penetration depth for 7 overlapping depths for all 5 films. Also shown is a linear fit for each film, indicating very little change in stress with increasing penetration depth. Excluding the $1.75 \mu \mathrm{m}$ film, the decrease averages $14 \mathrm{MPa}$ over the $0.25 \Rightarrow 2 \mu \mathrm{m}$ range. The error bars are \pm 5 $\mathrm{MPa}$, just larger than the marker size.

\section{Conclusions}

The data presented here do not show a significant stress variation with depth. The correction factors play a crucial role in proper derivation of the stress with depth. The depth-dependence of the unstrained a0 acts as a check on the data quality, and suggests that the correction is insufficient for the $1.75 \mu \mathrm{m}$ film. For absorbing films, such as gold, rotating the sample about the scattering vector of inclined planes is the most effective means of obtaining depth-dependent information. Although there are likely to be corrective factors to the results, judicious study of the materials constants and reflection directions should find those which are unaffected by the biaxial stress and can be used to remove the correction.

\section{E. Acknowledgments}

This work is supported by SSRL, which is funded by the U.S. Department of Energy (DOE), Office of Basic Energy Sciences under contract DE-AC03-76SF00515. Use of the Advanced Photon Source is supported by the DOE, BES-OER under Contract No. W-31-109-ENG-38. In addition, support from the Division of Materials Sciences of the Office of Basic Energy Sciences of the DOE (DE-FG03-89ER45387) is gratefully acknowledged.
[1] N.A. Fleck, G.M. Muller, M.F. Ashby and J.W. Hutchinson, Acta Metallurgica et Materialia 42, 475 (1994).

[2] W.D. Nix and H. Gao, Journal of the Mechanics and Physics of Solids 46, 411 (1998).

[3] J.S. Stolken and A.G. Evans, Acta Materialia 46, 5109 (1998).

[4] R. Venkatraman, P.R. Besser, J.C. Bravman and S. Brennan, Journal of Materials Research 9, 328 (1994).

[5] R.P. Vinci, E.M. Zielinski and J.C. Bravman, Thin Solid Films 262, 142 (1995).

[6] W.D. Nix, Metallurgical Transactions A (Physical Metallurgy and Materials Science) 20A, 2217 (1989).

[7] C.V. Thompson, Journal of Materials Research 8, 237 (1993).

[8] J.W. Hutchinson, Private Communication.

[9] M.L. Ovecoglu, D.M. Barnett and W.D. Nix, Acta Metallurgica 35, 1779 (1987).

[10] M.F. Doerner and S. Brennan, J. Appl. Phys. 63, 126 (1988).

[11] S.G.J. Mochrie, J. Appl. Cryst. 21, 1 (1988).

[12] W.R Busing, and H.A. Levy, Acta Cryst, 22, 457 (1967).

[13] B.M. Clemens and J.A. Bain, MRS Bulletin 17,46 (1992).

[14] M.F. Toney and S. Brennan, Phys. Rev. B 39, 7963 (1989). 